1999-03-19

\title{
Observations of lattice curvature near the interface of a deformed aluminium bicrystal
}

Brent L. Adams

b_I_adams@byu.edu

W. E. King

S. Sun

Follow this and additional works at: https://scholarsarchive.byu.edu/facpub

Part of the Mechanical Engineering Commons

\section{Original Publication Citation}

Philosophical Magazine A, 2, Vol. 8, No. 1, 9-25

\section{BYU ScholarsArchive Citation}

Adams, Brent L.; King, W. E.; and Sun, S., "Observations of lattice curvature near the interface of a deformed aluminium bicrystal" (1999). Faculty Publications. 625.

https://scholarsarchive.byu.edu/facpub/625 


\title{
Observations of lattice curvature near the interface of a deformed aluminium bicrystal
}

\author{
S. Sun, B. L ADAMS \\ Materials Science and Engineering, Carnegie-Mellon University, Pittsburgh, \\ Pennsylvania 15213-3890, USA \\ and W. E. KING \\ Chemistry and Materials Science Directorate, Lawrence Livermore National \\ Laboratory, Livermore, California, USA
}

\begin{abstract}
Reported here is a study of the pattern of lattice curvature near the interface of deformed high-purity aluminium (99.9999\%) bicrystals of specified crystallographic character (large-angle random). Curvature data are obtained from electron back-scattering diff raction pattern observations using orientation imaging microscopy. The concept of geometrically necessary dislocations (GNDs) is used as the central tool in the description of the observations. The samples studied were channel-die compressed perpendicular to the interface to plastic strain levels of 0.1 and 0.3 . At a strain level of 0.1 the primary observation is the development of a pile-up of GNDs (i.e. lattice curvature) near the interface. At the higher strain level of 0.3 , however, a dramatic change in the distribution is observed. The nature of this change suggests that the interface has absorbed (or emitted) some components of the nearby GND field, with an accompanying change in the local character of the interface towards a broader dispersion of misorientation character.
\end{abstract}

\section{§1. INTRODUCTION}

During plastic deformation, the constituent grains of polycrystals are observed to develop complex heterogeneous deformation patterns with variations in local strain and crystal orientation occurring over a wide range of length scales. Considerations of deformation heterogeneity are central to a deeper understanding of such phenomena as the Hall-Petch relationship, strain hardening in crystalline materials, and recrystallization and grain growth during recrystallization. The occurrence of this heterogeneity is not predicted by the classical theory of crystal plasticity (Taylor 1938) although the first-order features of crystallographic texturing are explained. Taylor's theory enforces only the mechanical compatibility of neighbouring grains during deformation and does not consider the equilibrium of mechanical forces across interfaces. It has been amply demonstrated by more recent finite-element simulations of polycrystalline plasticity (Harren et al. 1988, Becker 1991) that consideration of both compatibility and equilibrium naturally results in heterogeneous intragranular deformation patterns. There remains, however, a concern 
that consideration of these purely mechanical effects may not be a sufficient physical basis to predict reliably the complex patterns of heterogeneity that are observed. What is clearly missing is a consideration of the boundary itself, with its structure and (excess) free energy which must necessarily change with plastic deformation. It is anticipated that such considerations may help to establish the missing length scale in the theory of plasticity, the necessity of which is clearly indicated by the Hall-Petch relationship. The nature of this missing length scale has been discussed in the context of strain gradient plasticity by Fleck et al. (1994), and from the energetics perspective by Becker (1995).

Experimental evidence indicates that interfaces can resist the motion of lattice dislocations, or absorb or emit them depending upon the structure of the interface and local mechanical conditions. (An extensive literature review on the interactions of dislocations with grain boundaries and interfaces has been provided in the recent work of Sutton and Balluffi (1995).) These interactions, in connection with the aforementioned mechanical effects, will determine the patterns of lattice curvature observed in the vicinity of interfaces. The purpose of the study reported here is to describe observations of the fields of lattice curvature near the grain boundary in the deformed state of bicrystals of controlled crystallographic character (specified lattice orientations and boundary plane relative to the principal axes of channel die compression). (Comparisons of these observations with crystal-plastic finite-element simulations are described in the work of Sun et al. (1999).) Electron back-scattering pattern (EBSP) observations obtained by orientation imaging microscopy (OIM) were used to measure the local fields of lattice curvature. The methods employed are substantially the same as those described by Randle et al. (1996) for their characterization of the lattice rotation parameters in deformed polycrystalline samples. The main difference in approach is in the analysis of the data obtained. Whereas Randle et al. considered the spatial variation of a single parameter of misorientation 'distance' with respect to a fixed reference orientation, the present work considers the complete lattice curvature tensor, determined locally at each point in the field. The lattice curvature tensor can be used to obtain estimates of the second-order dislocation density tensor described by Nye (1953), Kröner (1958) and Bilby et al. (1958). Thus, a connection is made between the observations of the fields of lattice curvature and the geometrically necessary dislocations (GNDs) which support the observed curvatures.

The work of Randle et al. (1996) established congruence between the observations of misorientation and microstructural observations obtained by transmission electron microscopy (TEM). TEM observations of misorientation angles $\left(5-10^{\circ}\right)$ across cell block boundaries obtained by the Kikuchi technique (Liu 1994) compare well with the variations observed in neighbouring EBSPs in aluminium at strain levels comparable with those discussed here. The regularity of orientation perturbations observed in this study is consistent with the TEM observations of cell block boundaries and the cell walls found in their interiors (Barlow et al. 1985).

In $\S 2$ we review the fundamental equation of continuum dislocation theory, linking curvatures of the lattice and the elastic strain field with the dislocation tensor. A precise connection is established between lattice curvature and orientation measurements obtained from EBSPs. When the curvature of the elastic strain field is negligible compared with the lattice curvature, Nye's (1953) original formulation of the dislocation tensor is retrieved. It is in this context (i.e. in the absence of longrange stress fields) that we proceed in $\S 3$ to link the measured lattice curvatures with 
the GND content described by Ashby (1970). In order to make this connection we have opted to consider the lattice curvatures to be supported by the set of 24 pure edge and 12 pure screw dislocations associated with the $\{111\}\langle 110\rangle$ dislocation slip systems in fcc crystals. Using classical methods of linear programming (Dantzig 1963) we solve for the minimum density of dislocations required to support the observed lattice curvatures. GND distributions obtained following Nye's orthogonal deconstruction of the sc lattice are also compared with those supported on fcc lattices. In $\S 4$, bicrystalline samples which were strained by channel-die compression perpendicular to the interface, to two different height reductions, are discussed. The character of the bicrystalline interface is that of a random large-angle grain boundary. We also describe the determination of lattice curvatures by fine-scale OIM. The results are presented in $\S 5$ where densities of the GND distributions are depicted along two directions with respect to the bicrystalline interface. GND densities adjacent to the interface with those removed some distance are compared. It is found that GNDs mainly concentrate near the interface, and that their spatial distributions vary with strain level. Examination of the distributions suggests that the role of the bicrystalline boundary varies with strain level, and with the evolution of the crystallographic character of the interface.

\section{§ 2. BASIC RELATIONSHIPS}

Here we briefly review the main ideas behind the fundamental equation of continuum dislocation theory. First, consider the continuity condition on the displacement field $\mathbf{u}(\mathbf{x})$. (The displacement fields are defined only in an infinitesimal neighbourhood of position $\mathbf{x}$. For a more complete discussion the reader is referred to the book by Kröner (1958).) Around a closed curve, continuity demands that

$$
{ }_{C} \mathrm{du}={ }_{C}^{\dagger} \beta \mathrm{d} \mathbf{x}=0,
$$

where $\beta$ is the distortion tensor defined by $\mathrm{d} u_{i}=\beta_{j i} \mathrm{~d} x_{j}$. After separation of the distortion tensor into its elastic and plastic parts: $\beta=\beta^{\mathrm{e}}+\beta^{\mathrm{p}}$, application of Stokes' theorem to equation (1) obtains the expression:

$$
\oiint_{S} \operatorname{curl}\left(\beta^{\mathrm{e}}+\beta^{\mathrm{p}}\right) \mathrm{d} \mathbf{s}=0,
$$

where $S$ is any continuous surface patch bounded by $C$ and ds denotes the outward normal associated with an element of the surface patch, weighted by its surface area. Since equation (2) must be valid at any point in the crystal, and for any closed curve $C$, it follows that the plastic and elastic curvatures must be related by

$$
\operatorname{curl} \beta^{\mathrm{e}}=-\operatorname{curl} \beta^{\mathrm{p}} \text {. }
$$

The net Burgers vector $\mathbf{B}$, associated with the Burgers circuit $C$, is defined in connection with the dislocation tensor $\alpha$ by the expression

$$
\mathbf{B}=\oint_{S} \alpha \mathrm{d} \mathbf{s}=-\oint_{S} \operatorname{curl} \beta^{p} \mathrm{~d} \mathbf{s}
$$

Comparing equations (2), (3) and (4), it is evident that the dislocation tensor can be defined in terms of the curvature of the elastic distortion tensor:

$$
\alpha=\operatorname{curl} \beta^{\mathrm{e}} .
$$


Equation (5) is called the fundamental equation of continuum dislocation theory.

We next proceed to establish the connection between the dislocation tensor, the lattice curvature tensor and lattice misorientation. It is clear from the definition of the elastic distortion tensor that the following connection exists between the infinitesimal elastic strain tensor $\varepsilon$ and the rotation tensor $\omega$ (expressed here in component form for additional clarity):

$$
\alpha_{i j}=e_{i k l} \beta_{l j, k}^{\mathrm{e}}=e_{i k l}\left(\varepsilon_{j l, k}^{e}+\omega_{j l, k}^{\mathrm{e}}\right) .
$$

Here $e_{i k l}$ denotes components of the permutation tensor. $\omega$ is related to the (small rigid-body) rotations of the lattice. It is customary to express finite rotations in terms of a related tensor $\mathbf{g}$, the rotation of which is taken in a right-handed sense by an angle $\theta$, about the direction $\hat{\mathbf{m}}$ according to the formula

$$
g_{i j}=\delta_{i j} \cos \theta-e_{i j k} m_{k} \sin \theta+(1-\cos \theta) m_{i} m_{j} .
$$

In the present context it can be assumed that $\theta \ll 1$, in which case equation (7) is adequately approximated by

$$
g_{i j}=\delta_{i j}-e_{i j k} \theta m_{k} .
$$

The relationship between the tensor $\mathbf{g}$ (in its small angle form) and $\omega$ is just

$$
\omega_{i j}^{\mathrm{e}}=g_{i j}-\delta_{i j} \text {. }
$$

Thus

$$
\alpha_{i j}=e_{i k l}\left(\varepsilon_{j l, k}^{\mathrm{e}}+g_{j l, k}\right) .
$$

For our purposes here, equation (10) is the most convenient form of the fundamental equation.

Other expressions for the fundamental equation make use of the lattice curvature tensor $\kappa$. The lattice curvature tensor represents the lattice orientation change $\mathrm{d} \phi_{i}$ along $\mathrm{d} x_{j}$ according to the relation

$$
\mathrm{d} \phi_{i}=\kappa_{i j} \mathrm{~d} x_{j}=\phi_{i, j} \mathrm{~d} x_{j} .
$$

The physical meaning of the components of the curvature tensor is that the diagonal components of $\kappa_{i j}$ are twistings about the $x_{i}$ axes and the off-diagonal terms are bendings of the $x_{i}$ plane about the $x_{j}$ direction. It is then clear that $\phi_{i}$ is identified with $\theta m_{i}$ in equation (8), and $\mathrm{d} \phi_{i}=\mathrm{d} \theta m_{i}$. When these definitions are used, two additional forms of the fundamental equation can be stated:

$$
\kappa_{i j}=-\alpha_{i j}+\frac{1}{2} \delta_{i j} \alpha_{k k}-e_{i j k} \varepsilon_{j k, l}^{\mathrm{e}}
$$

and

$$
\alpha_{i j}=-\kappa_{i j}+\delta_{i j} \kappa_{p p}-e_{i k l} \varepsilon_{j l k}^{\mathrm{e}} .
$$

The physical meaning of equations (10), (12) and (13) is that lattice curvature obtains from two sources: gradients in the elastic strain tensor, and the presence of dislocation networks which possess a net Burgers vector. In the absence of long-range elastic stress fields the term containing the elastic strain tensor in equations (10), (12) and (13) vanishes and we retrieve the original results of Nye (1953).

Equation (10) establishes a close connection with gradients in the local lattice orientation, or the presence of misorientations in the orientation field $\mathbf{g}(\mathbf{x})$ over small variations in position $\mathrm{dx}$. Experiments are limited in the magnitude $|\mathrm{d} \mathbf{x}|$ over which 
distinctive measurements of orientation, g, can be obtained. With respect to EBSPs, the limiting spatial resolution is dependent upon the accelerating voltage of the electron microscope, the atomic-number of the material, and other factors (Adams et al. 1993). Typical resolution limits are about $100 \mathrm{~nm}$ in metal and ceramic alloys.

\section{§3. GEOMETRICALLY NECESSARY DiSLOCATIONS}

To the extent that Nye's approximation holds, the fundamental relation can be expressed in the following form:

$$
\alpha_{i j}=e_{i k l} g_{j l, k} .
$$

Thus, a connection is made between experiments (fine-scale OIM, based upon EBSPs) and the dislocation tensor field $\alpha(\mathbf{x})$. What is necessary is the capability to measure the components of the curvature of the orientation field.

As was shown by Nye (1953), there exists a precise connection between the dislocation tensor and the network of dislocations in the neighbourhood of the point in question. The relationship can be expressed as

$$
\boldsymbol{\alpha}=\sum_{s=1}^{S} \rho^{(s)} \mathbf{b}^{(s)} \otimes \hat{\mathbf{z}}^{(s)},
$$

where the sum is over the types of dislocation present in the neighbourhood of the point, $\rho^{(s)}$ denotes the density of dislocations of type $s, \mathbf{b}^{(s)}$ is the Burgers vector of that type, and $\hat{\mathbf{z}}^{(s)}$ means the line direction of the same. The dyadic $\mathbf{b}^{(s)} \otimes \hat{\mathbf{z}}^{(s)}$ provides a geometrical definition of dislocation $s$. We shall further presume that $s$ enumerates the set of all possible dislocations which may be present in the crystal. This set we shall denote as $\Delta$, where

$$
\Delta=\left\{\mathbf{b}^{(1)} \otimes \mathbf{z}^{(1)}, \ldots, \mathbf{b}^{(s)} \otimes \mathbf{z}^{(s)}, \ldots, \mathbf{b}^{S} \otimes \mathbf{z}^{S}\right\} .
$$

Dislocations which give rise to lattice curvature are identified as 'geometrically necessary' by Ashby (1970). Thus, we think of $\alpha(\mathbf{x})$ as describing the field of GNDs.

If the types and densities of GNDs present are known, the dislocation tensor is also known through equation (15). The converse of this expression is not true, however. For example, if it is presumed that $\alpha$ is known from measurements of the gradient of lattice orientation, according to equation (14), then it is not generally possible to retrieve the dislocation densities; this is true even if information about the set $\Delta$ is known. The reason is that there generally would exist many combinations of Burgers vectors and line directions which can support an arbitrary dislocation tensor; thus, an infinite variety of network types can support the same lattice curvature. The exception is when the set $\Delta$ contains only $S=9$ elements. In this case, assuming that the elements of $\Delta$ form a complete basis for the linear space of second-order tensors (to which $\alpha$ belongs), then a linear system of equations of the form

$$
\alpha_{i j}=\sum_{s=1}^{9} \rho^{(s)} b_{i}^{(s)} z_{j}^{(s)}
$$

obtains which can be solved for $\rho^{(s)}$. Such is the case for the example first considered by Nye (1953) where nine dislocations having Burgers vectors of the type $\mathbf{b}^{(s)} / /\langle 100\rangle$ and also $\hat{\mathbf{z}}^{(s)} / /\langle 100\rangle$ were considered. This example corresponds to a sc crystal lattice dislocated by three pure screw and six pure edge dislocations. In this case, only one dislocation from the set $\Delta$ contributes to each component of $\alpha$. Thus a one-to-one 
correspondence between the components of $\alpha$ and the dislocations in $\Delta$ is established. We shall refer to this example as the sc deconstruction or the orthogonal deconstruction of the dislocation tensor.

For our purposes here we prefer to consider a set $\Delta$ which corresponds to the $\{111\}\langle 110\rangle$ slip systems in fcc crystals. Slip of this type can be represented by the motions of a set of 12 pure screw dislocations whose Burgers vectors and line directions lie in the $\langle 110\rangle$ directions, and by a set of 24 pure edge dislocations whose Burgers vectors lie in $\langle 110\rangle$ directions and whose line directions lie along $\langle 112\rangle$. In this case, which we shall refer to as the fcc deconstruction of the dislocation tensor; no unique solution for the dislocation densities exists. What can be considered, however, is the particular set of nine dislocation types, taken from among the 36 which are available, for which a 'lower bound' on the total dislocation density can be obtained.

A lower-bound fcc deconstruction is calculated from classical methods of linear programming(here using the Simplex method (Dantzig 1963)) by coupling equation (15) with the following constraint:

$$
\operatorname{minimum}\left\{\rho_{\mathrm{GND}}=\sum_{s \in \Delta^{\prime}} \rho^{(s)} \mid \Delta^{\prime} \subset \Delta, \boldsymbol{\alpha}=\sum_{s \in \Delta^{\prime}} \rho^{(s)} \mathbf{b}^{(s)} \otimes \mathbf{z}^{(s)}\right\} .
$$

Stated in words, we seek to find the subset $\Delta^{\prime}$ of $\Delta$ for which the set of densities satisfies the local lattice curvature, and which minimizes the total required dislocation density $\rho_{\mathrm{GND}}$. (Here $\rho_{\mathrm{GND}}$ has been used to emphasize the connection with GNDs.) Linear programming solutions do not guarantee the uniqueness of $\Delta^{\prime}$, but they do ensure the lower-bound quality of $\rho_{\mathrm{GND}}$. (Note that the upper bound on $\rho_{\mathrm{GND}}$ is infinite.)

\section{§ 4. EXPERIMENTAL DETERMINATION OF THE GEOMETR ICALLY NECESSARY \\ DISLOCATION DENSITY IN DEFORMED BICRYSTALS}

The samples considered were $99.9999 \%$ pure aluminium bicrystals, prepared by ultrahigh-vacuum diffusion bonding of lapped and polished interfaces (King 1991). Samples of identical starting configuration were strained by channel-die compression to height reductions of $10 \%$ and $30 \%$. The starting configuration of the bicrystal, its crystallographic orientations, and the direction of compression are shown in figure 1. In crystal A the $X, Y$ and $Z$ axes correspond to the crystallographic directions [001], [110] and [110] respectively. In crystal B the same sample axes coincide with directions [110], [332] and [113] respectively. The calculated Taylor factors in this configuration are 3.88 for crystal A and 3.02 for crystal B.

Deformed samples were sectioned along planes containing the compression direction and parallel to the free end of the sample. After polishing and electropolishing, OIM was used to measure the lattice orientation (resolution limit approximately $1^{\circ}$ ) at many points about (100 000) lying along numerous lines (about 300) perpendicular to the bicrystalline interface (parallel to the compression axis). The main results were obtained from scans taken only near the central region $( \pm 400 \mu \mathrm{m})$ of the bicrystal in order to minimize the effects of external surfaces, friction effects, etc. The separation between measurement points was selected to be $2.5 \mu \mathrm{m}$ after preliminary investigation revealed negligible levels of orientation difference at finer scales of inquiry. Analysis of the experimental data confirmed the hypothesis that lattice curvatures (tilts and twists) about directions perpendicular to the compression 

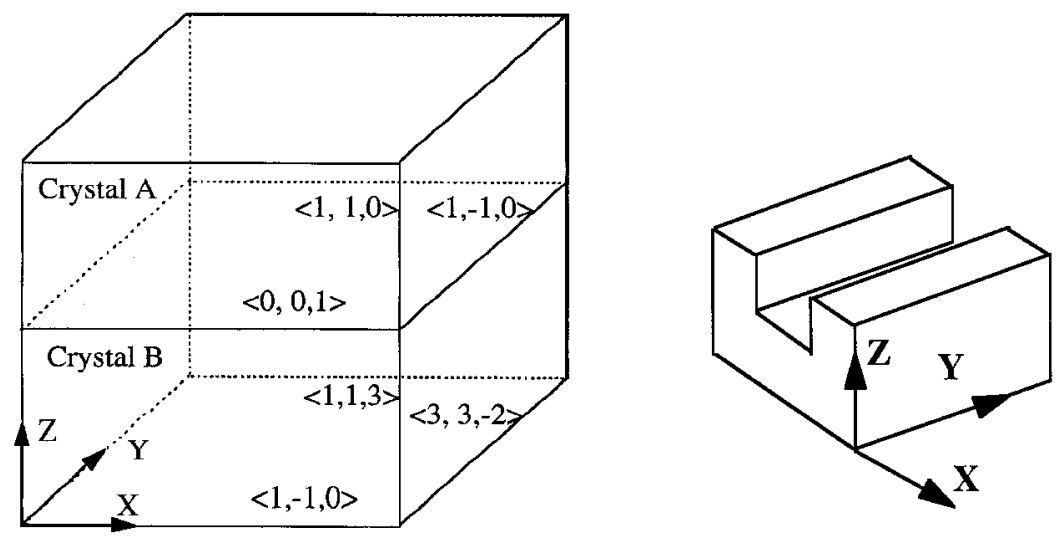

Figure 1. Orientations of the two constituents crystals in relation to the channel die and the compression direction $Z$.

axis are smaller (by factors of about four) than those about the compression direction in these samples. Thus, the main contribution to the dislocation tensor comes from nine of the 27 components of $g_{j l k}$ appearing in equation (14), that is those with derivatives in the $Z$ direction. This observation is in accordance with our expectations, given the geometry of the bicrystal and the imposed boundary conditions. Since data were obtained from only a single section plane (the $X-Z$ plane) the orientation of the derivatives with respect to the direction $Y$ were not available; these derivatives have been set equal to zero in the analysis for the dislocation density.

The curvature data was averaged in the following way. Directions $X, Z$ lie in the section plane, with $X$ lying parallel to the bicrystalline boundary and $Z$ lying parallel to the compression axis. Orientation data were obtained on a square array aligned with $X, Z$. At every point possible, the components of the orientation curvature were obtained. Thus, $g_{j l, k}(x, z)$ was obtained for all possible $x, z$ points. The data were then averaged over $x$ according to the relation

$$
\tilde{g}_{j l, k}(z)=\frac{1}{L} \int_{x_{0}}^{x_{0}+L} g_{j l, k}(x, z) \mathrm{d} x
$$

and averaged over $z$ according to the relation

$$
\tilde{g}_{j l, k}(x)=\frac{1}{L} \int_{z_{0}}^{z_{0}+L} g_{j l, k}(x, z) \mathrm{d} z .
$$

These averaged orientation curvatures were then used in connection with equation (14) to estimate the components of the dislocation tensor. The Simplex method in standard tableau form was used to solve equation (18) for lower-bound estimates on the GND density $\rho_{\mathrm{GND}}$.

\section{§5. DesCRIPTION OF THE RESUlts}

Figures 2 and 3 delineate the GND distributions at the two strain levels along the direction $Z$, obtained from orientation curvatures averaged according to equation (19). Figures 4 and 5 portray the GND configurations along direction $X$, as calculated from orientation curvatures averaged according to equation (20). It is clear that 


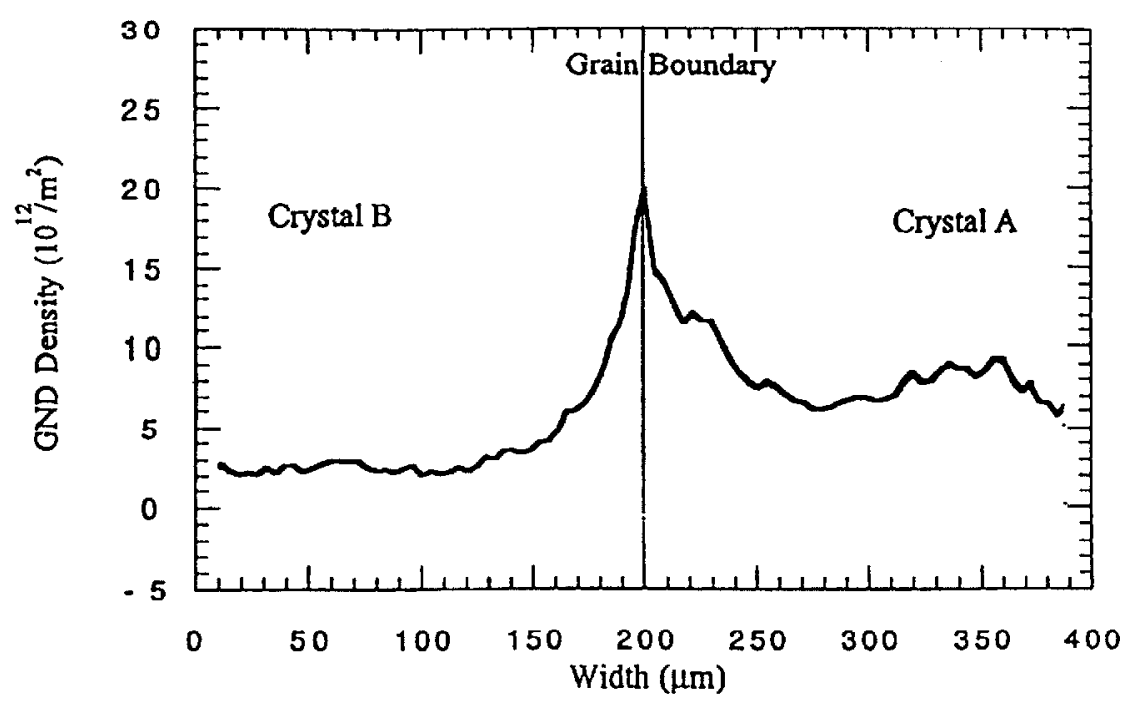

Figure 2. GND distribution in the $10 \%$ strained aluminium bicrystal (along direction $Z$ ).

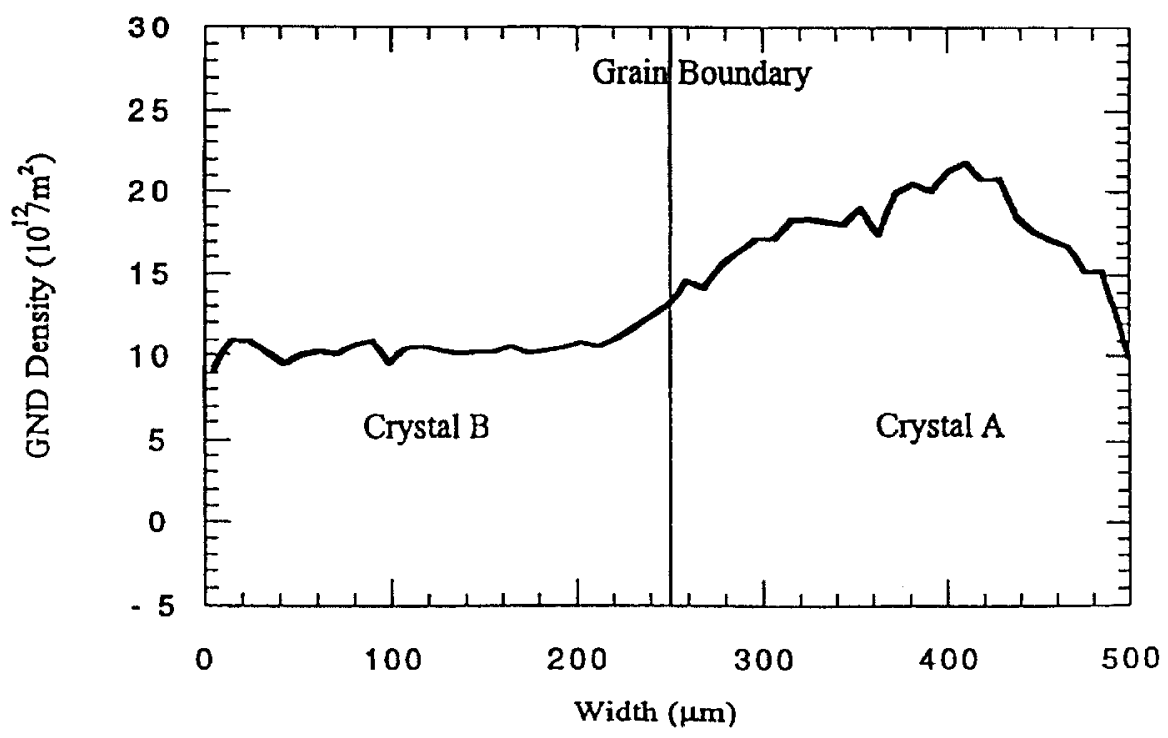

Figure 3. GND distribution in the $30 \%$ strained aluminium bicrystal (along direction $Z$ ).

the GND distributions vary substantially along the direction $Z$, while they are much more uniform along the direction $X$. The main feature at a strain level of 0.1 (figure 2 ) is a sharp peak at the grain boundary with a peak half-width of approximately $50 \mu \mathrm{m}$. The plateau levels adjacent the main peak are higher in crystal A, which also has the higher Taylor factor. Also, a small broad secondary peak is situated in crystal A centred approximately $150 \mu \mathrm{m}$ from the interface.

At a strain level of 0.3 (figure 3) there is no longer any evidence of the GND peak at the interface, but a large broad peak is observed in crystal A centred at approxi- 


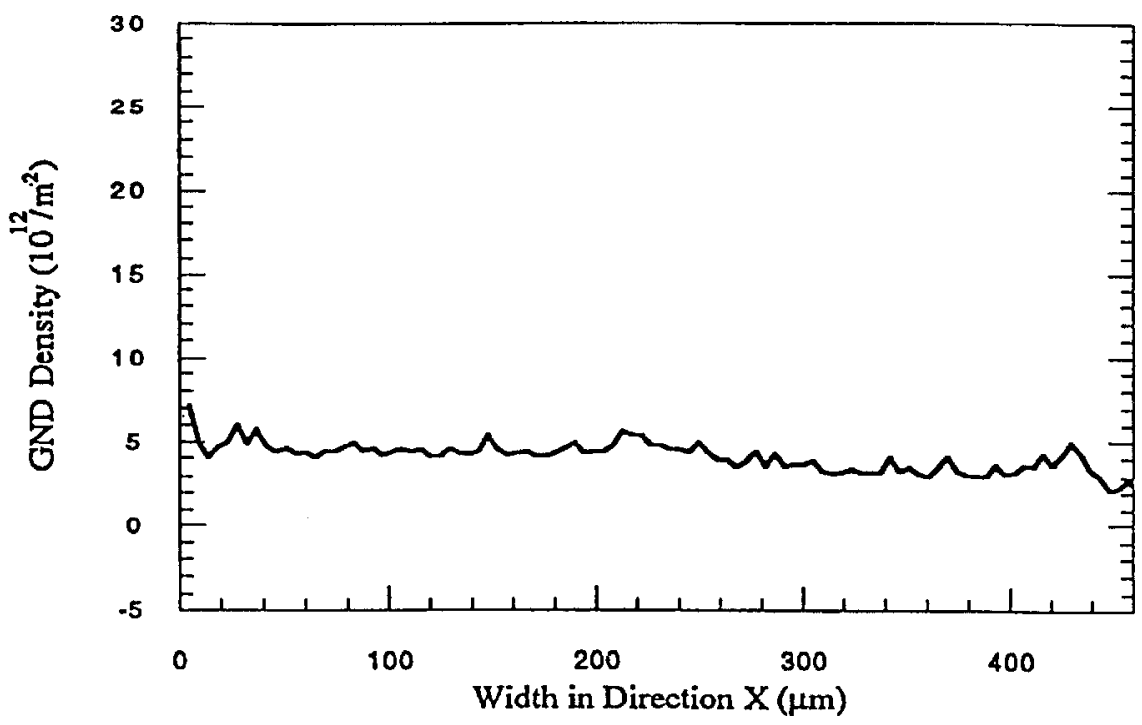

Figure 4. GND distribution in the $10 \%$ strained aluminium bicrystal (along direction $X$ ).

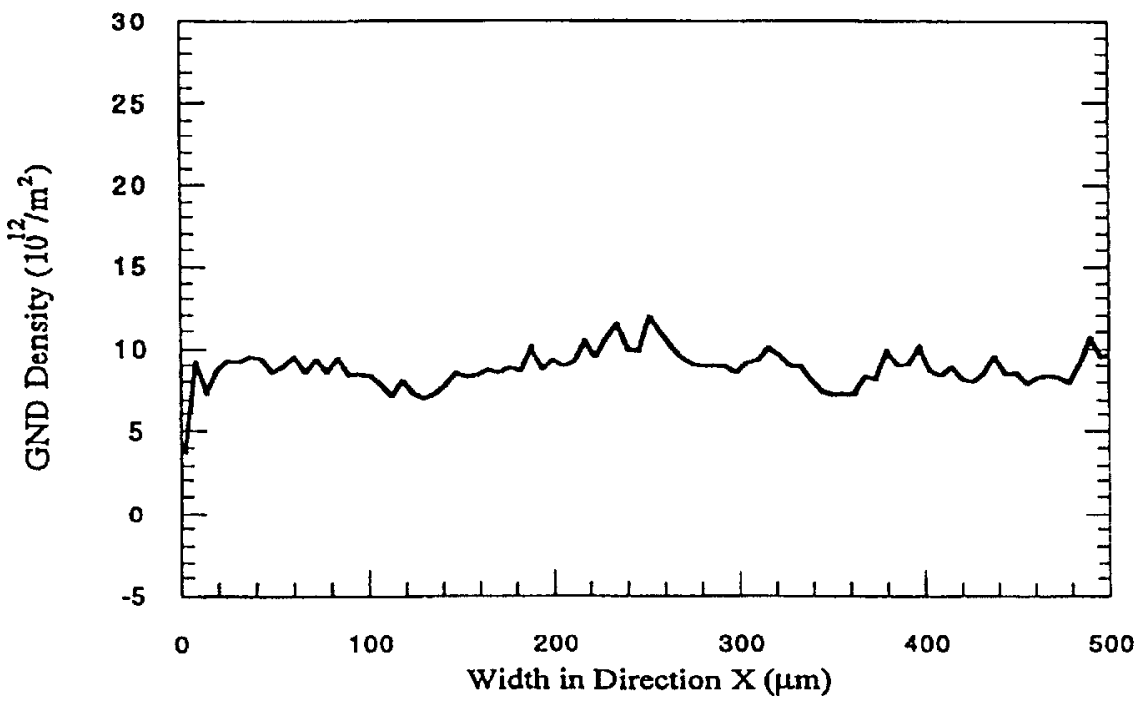

Figure 5. GND distribution in the $30 \%$ strained aluminium bicrystal (along direction $X$ ).

mately $150 \mu \mathrm{m}$ from the interface. This peak corresponds in location and breadth with the secondary peak observed at the lower strain level. The plateau level in crystal B is now at a level of approximately $10^{13} \mathrm{~m}^{-2}$. Distributions of the average GND density along the $X$ direction are nominally level and have values of approximately $5 \times 10^{12} \mathrm{~m}^{-2}$ at a strain level of 0.1 (figure 4 ) and $10^{13} \mathrm{~m}^{-2}$ at a strain of 0.3 (figure 5).

In previous work, the deformation field far away from grain boundaries was observed to be much more homogeneous (Barlow et al. 1985), presumably because 
of weaker influence from the interface. Figures 6 and 7 show the average GND distributions along the $Z$ direction inside the two constituent crystals measured at a distance of $850 \mu \mathrm{m}$ away from the bicrystalline boundary. In both crystal A and crystal B the GND density levels are found to be less than $4 \times 10^{12} \mathrm{~m}^{-2}$ at both strain levels, with the observed levels being slightly higher in crystal A (which has the higher Taylor factor).

Comparisons of the estimated GND density fields obtained from Nye's sc deconstruction are compared with the lower-bound fec deconstruction in figures 8 and 9

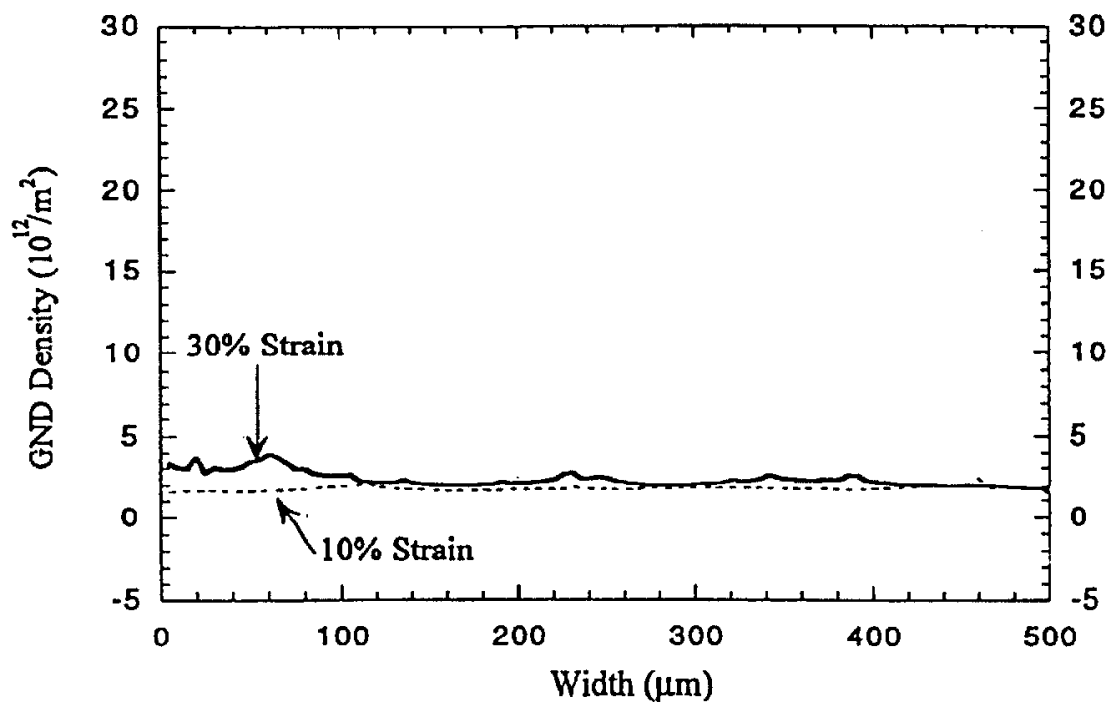

Figure 6. GND distribution removed $850 \mu \mathrm{m}$ from the interface in crystal B

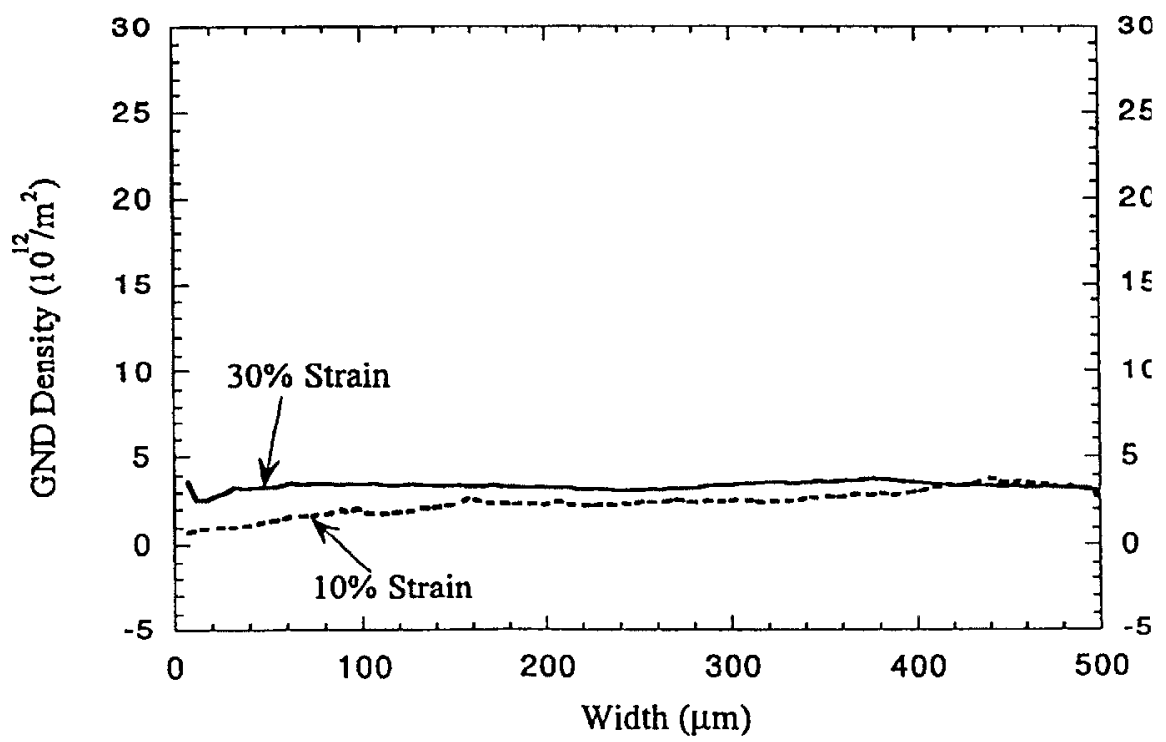

Figure 7. GND distribution removed $850 \mu \mathrm{m}$ from the interface in crystal A. 
for the two strain levels. The spatial distributions of GND densities are clearly similar in shape, but the densities obtained from the lower-bound fcc deconstruction are always higher than those obtained by the sc deconstruction. This is consistent with the observation that more dislocations should be required to support the observed deformation gradients when dislocations are restricted to the available (non-orthogonal) $\{111\}\langle 110\rangle$ slip systems in fec crystals. The difference between the two deconstructions is observed to increase with increasing strain level.

In order to facilitate a deeper insight into the changes that occur in the GND distribution as a function of strain it is instructive to construct the ratio $\Psi(z)$ of

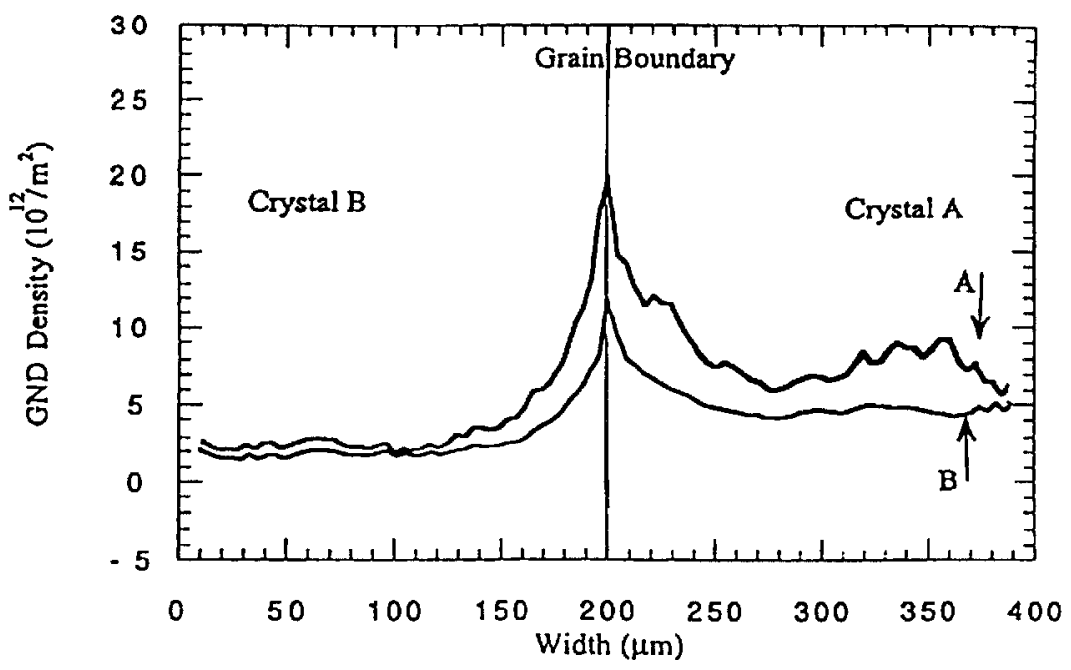

Figure 8. GND distribution at $10 \%$ plastic strain estimated by the sc deconstruction (curve B) and by the lower-bound fcc deconstruction (curve A).

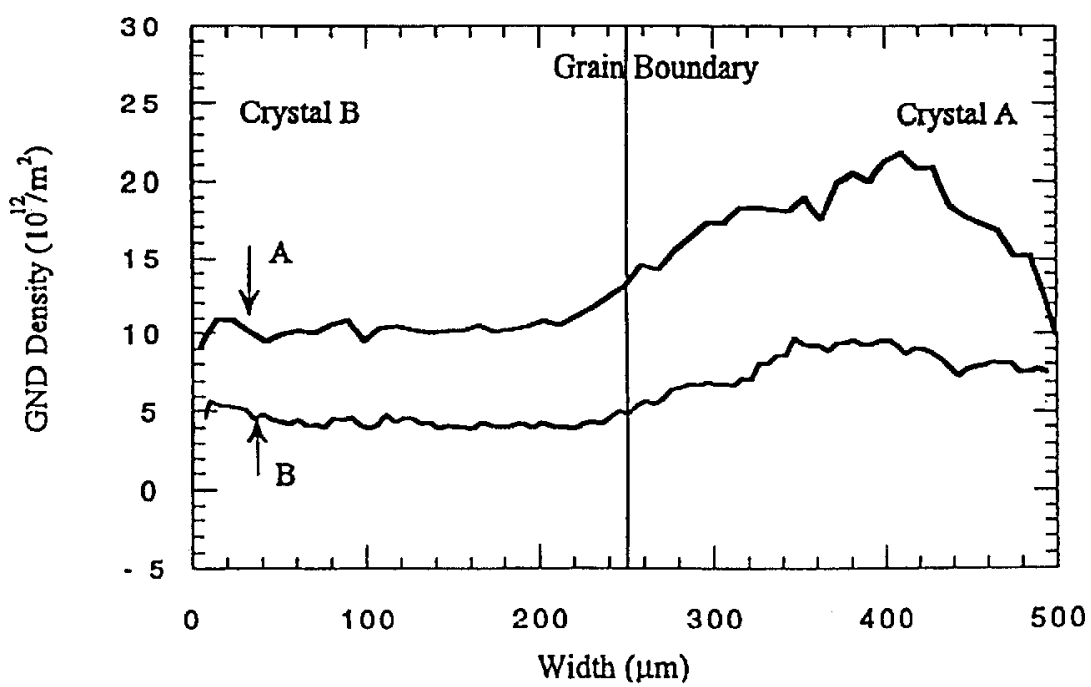

Figure 9. GND distributions at 30\% plastic strain estimated by the sc deconstruction (curve B) and by the lower-bound fce deconstruction (curve A). 
components of the GND density with Burgers vectors lying in the bicrystalline plane, relative to the total. (The reader should note that if the Burgers vectors were randomly directed, then we should expect $\Psi(z)=\frac{2}{3}$.) Figures 10 and 11 show the distribution of $\Psi(z)$ at the two different strain levels. It is evident that a higher proportion of Burgers vector lying in the interface plane is present in crystal B relative to crystal A at both strain levels. At the higher strain level, this difference has become even more exaggerated, suggesting that plastic deformation in excess of 0.1 has resulted in a reduction in $\Psi(z)$ near the interface in crystal A, but an increase near the interface in crystal B. In particular, the peak levels of $\Psi(z)$ have shifted from approximately $15 \mu \mathrm{m}$ away from the boundary in crystal B at a strain of 0.1 to approximately $50 \mu \mathrm{m}$ at a strain of 0.3 . In crystal A a similar shift in the minimum level of $\Psi(z)$ is observed.

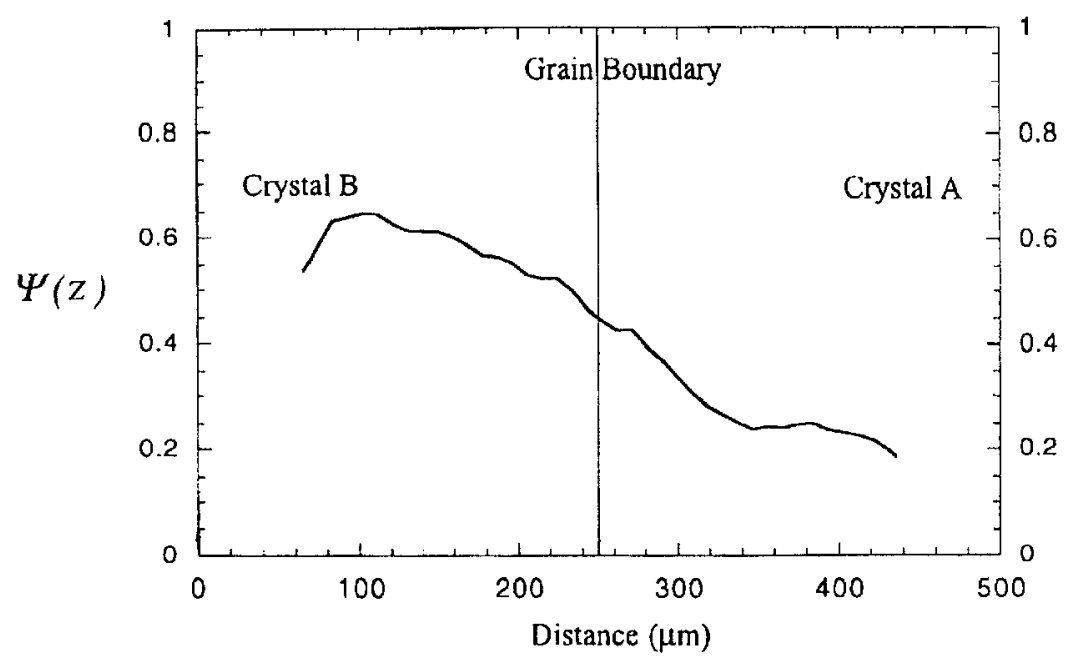

Figure 10. Distribution of $\Psi(z)$ at $10 \%$ plastic strain.

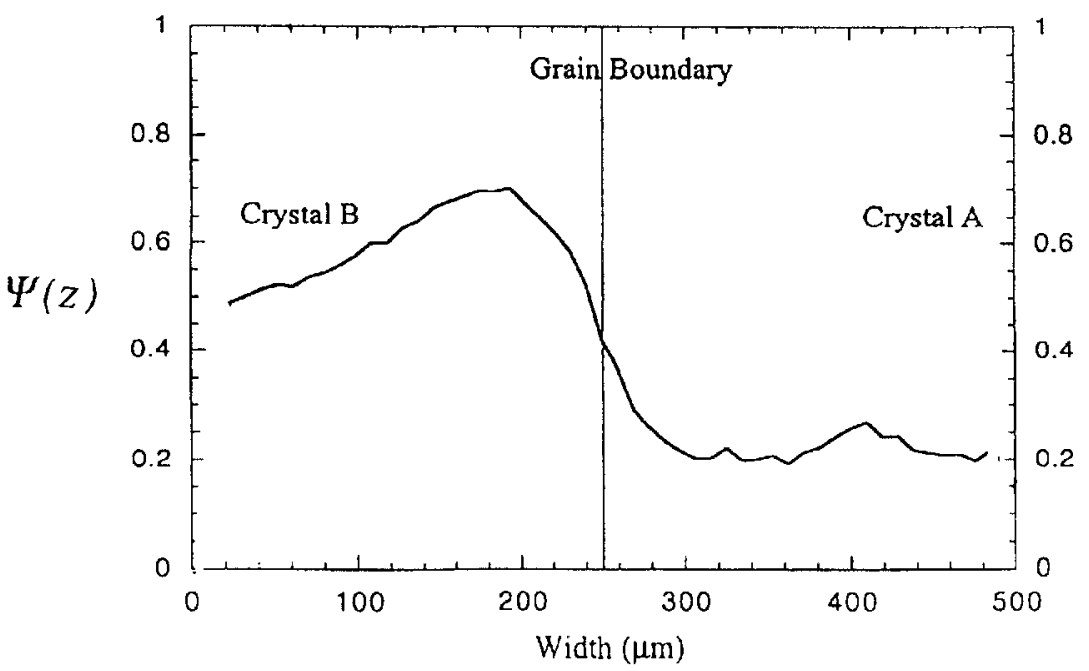

Figure 11. Distribution of $\Psi(z)$ at $30 \%$ plastic strain. 
It is also instructive to examine the individual components of the dislocation tensor as estimated from equation (14), and as expressed in the coordinate system of the bicrystal (i.e. $X, Y$ and $Z$ ). The reader should note from the definition of the dislocation tensor (left-hand side of equation (4)) that the left index of $\alpha$ (index $j$ in $\alpha_{j k}$ ) denotes the direction of the Burgers vector, and the right index (index $k$ in $\alpha_{j k}$ ) specifies the line direction. Here we shall let the index 3 denote directions parallel to $Z$, index 2 is identified with $Y$ and index 1 with direction $X$ in the bicrystal. Here we concentrate on two representative components of the dislocation tensor. Figures 12 and 13 show the variations in $\alpha_{22}$ and $\alpha_{23}$ as a function of distance $z$ in the $Z$ direction of the bicrystal. $\alpha_{22}$ denotes the component of the GND distribution

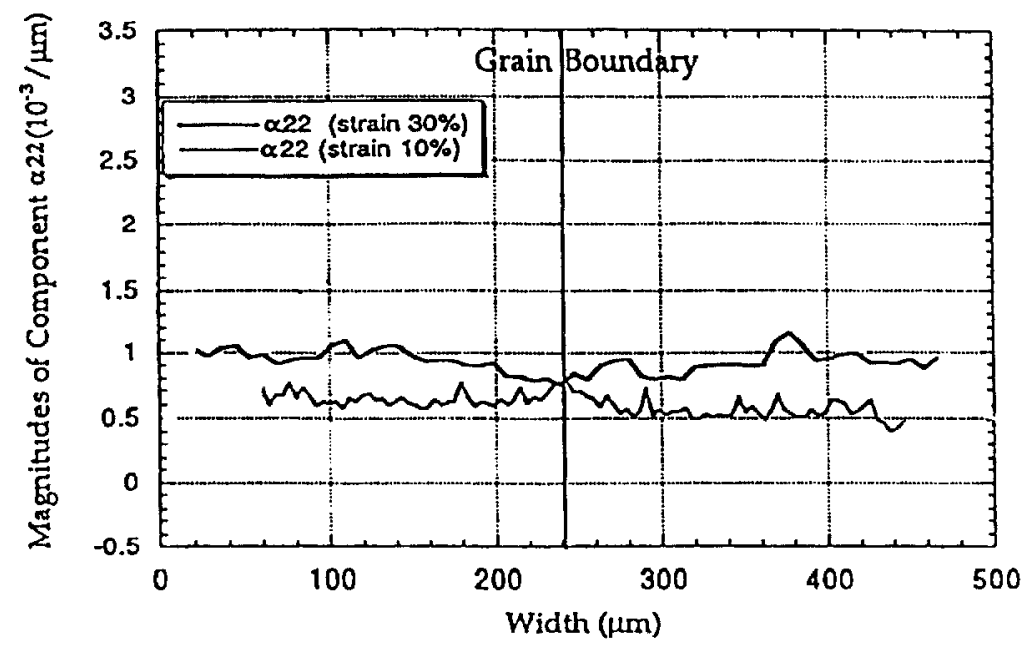

Figure 12. Distribution of $\alpha_{22}(z)$ at plastic strain levels of 0.1 (lower curve) and 0.3 (higher curve).

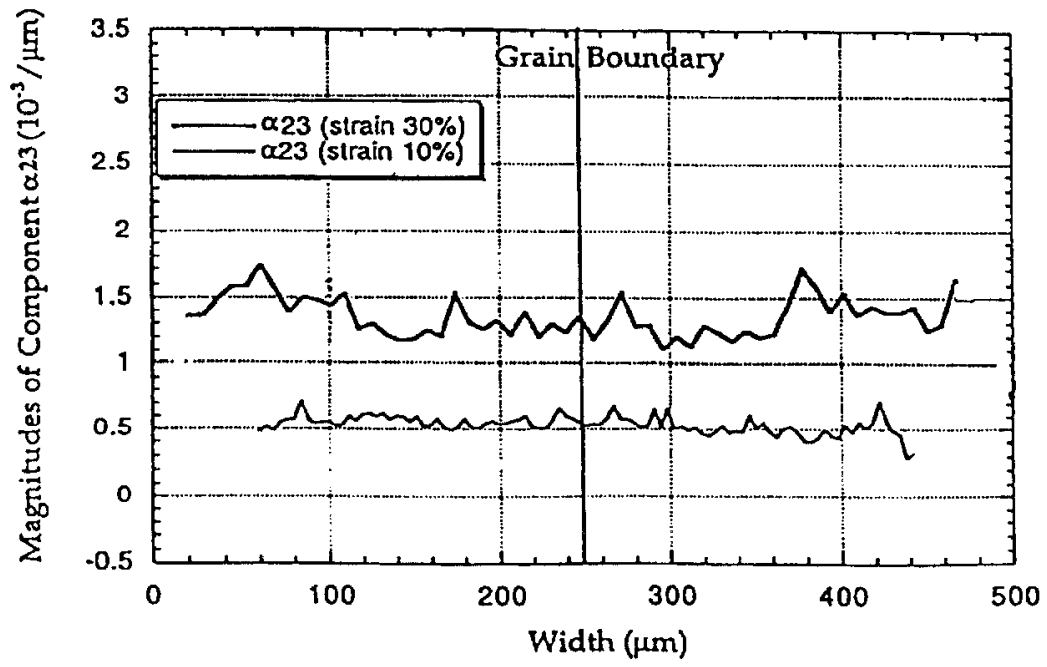

Figure 13. Distribution of $\alpha_{23}(z)$ at plastic strain levels of 0.1 (lower curve) and 0.3 (higher curve). 
supported by dislocations of screw type with their line and Burgers directions along $Y . \alpha_{23}$ is associated with the component of the GND distribution supported by edge dislocations with their line directions along $Z$ and their Burgers vectors along $Y$. Although the details and magnitudes of the distributions of various components vary somewhat, the qualitative behaviour of $\alpha_{22}$ is similar to what is observed for $\alpha_{11}, \alpha_{12}$, $\alpha_{21}, a_{31}$ and $a_{32}$. The behaviour of $\alpha_{23}$ is similar to what is observed for components $\alpha_{13}$ and $\alpha_{33}$. The concave downward trend for $\alpha_{22}$ at a strain level of 0.3 and the slight concave upward trend at a strain level of 0.1 is also a common feature among similar components (figure 12). Concavity is less noticeable with $\alpha_{23}$ and its similar subset (figure 13). The main difference to be noted is that components of the dislocation tensor with their line directions perpendicular to the interface (right index at 3 ) increase more with increasing strain than do components with line directions lying in the plane of the interface.

Further insight obtains by considering the distribution of misorientations associated with pairs of points placed at equidistant positions perpendicular to the interface. Two distances were chosen: 'near-field' misorientations for pairs of points lying at $2-5 \mu \mathrm{m}$ from the interface plane, and 'far-field' misorientations taken at distances about $100 \mu \mathrm{m}$ from the interface. Numerous pairs of points were considered in each case. The results are expressed as a dispersion of lattice misorientation in the Rodrigues-Frank representation of the fundamental zone of misorientation (Sutton and Balluffi 1995). The results at 0.3 plastic strain are highlighted here. Figures 14 and 15 show the far-field and near-field dispersions respectively. It is evident that the dispersion is much broader near the interface. The near-field dispersion overlaps with the $\Sigma=15$ coincidence site lattice relationship (about $20 \%$ of

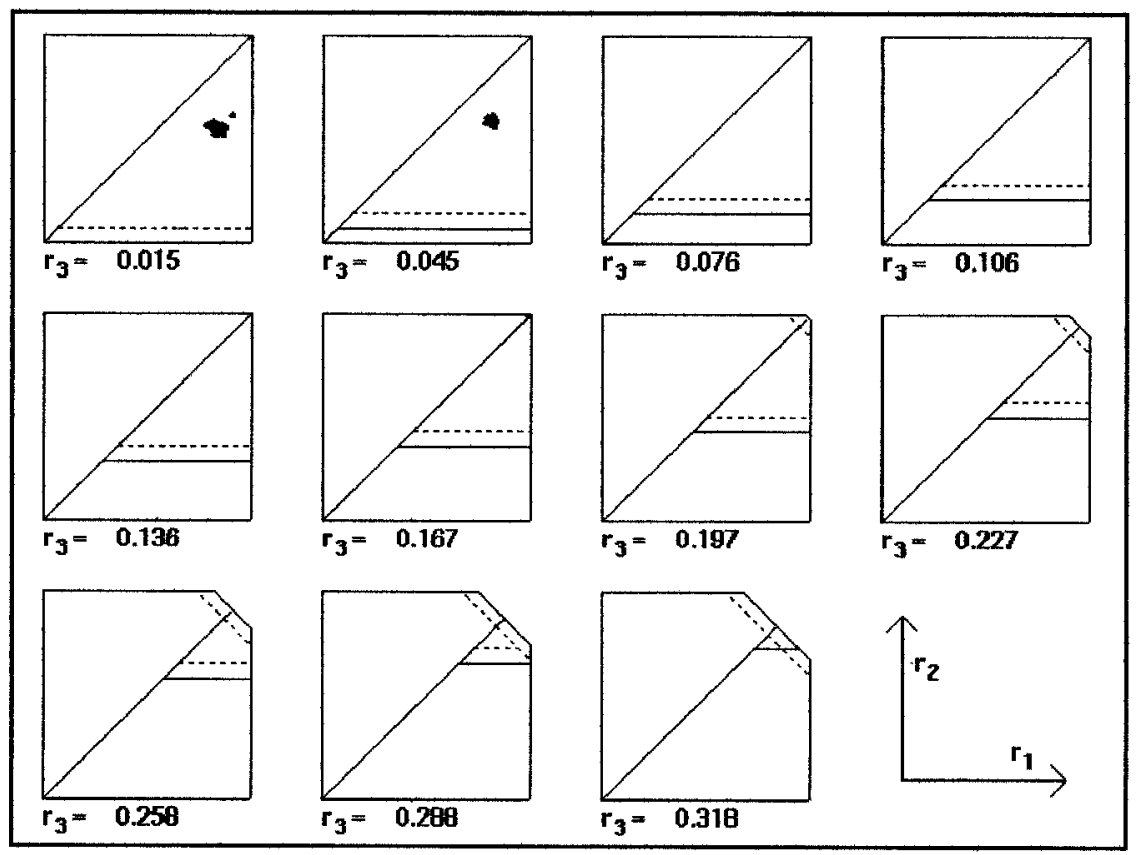

Figure 14. Far-field distribution of interface misorientation at a distance of $100 \mu \mathrm{m}$ from the interface at 0.3 plastic strain. 


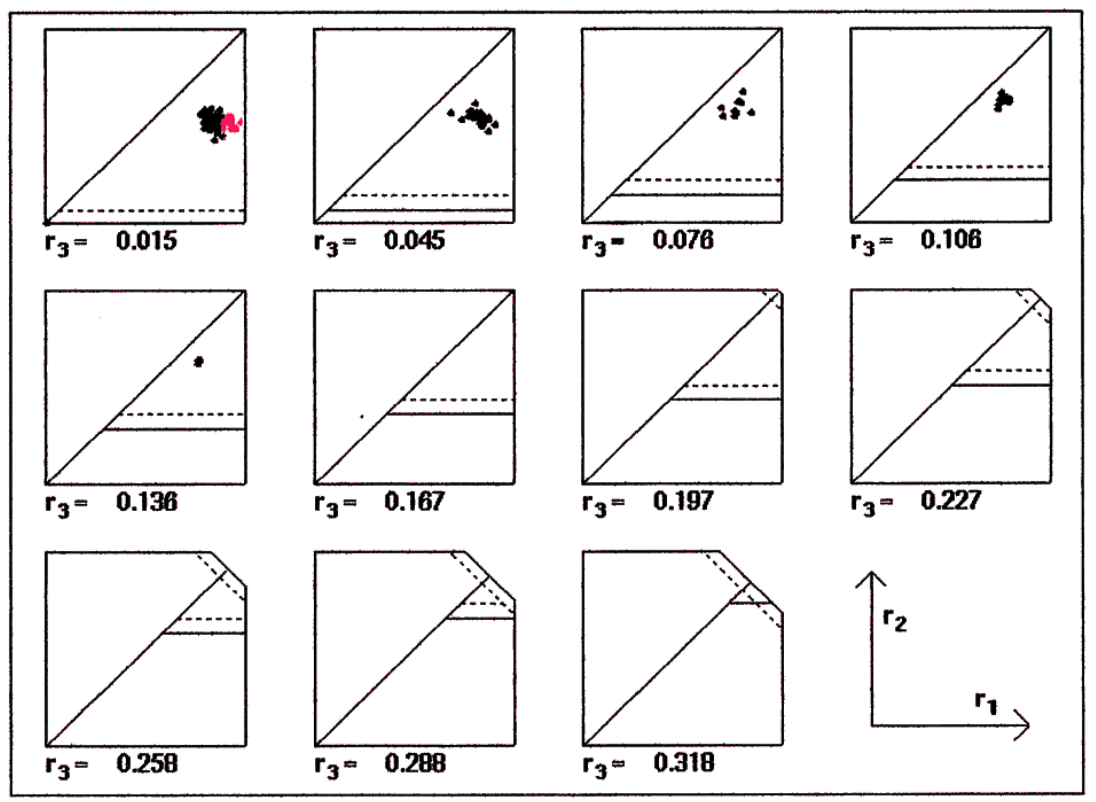

Figure 15. Near-field dispersion of interface misorientation at a distance of $2-5 \mu \mathrm{m}$ from the interface at 0.3 plastic strain (red points satisfy Brandon's criterion for $\Sigma=15$ ).

the point pairs), in accordance with Brandon's (1996) criterion. The initially flat interface has been roughened by the deformation field and is observed to have developed undulations at a wavelength of approximately $10 \mu \mathrm{m}$. It is presumed that this change in local character and dispersion derives as a result of the absorption or emission of dislocations into the interface during plastic deformation. That such processes must occur as strain levels increase is strongly suggested by figures 2,3 and $10-13$.

\section{$\S 6$. Discussion}

The main observation is that the dislocated state of the crystal lattice near the interface of a deformed aluminium bicrystal is strongly dependent upon the magnitude of the strain. At a level of 0.1 plastic strain the GND distribution in the vicinity of the interface is observed to peak at the boundary, much like one would expect from the trapping of dislocation pile-ups. Notably, the breadth of the peak (about $200 \mu \mathrm{m}$ ) is much larger than the width of the deformed zone commonly observed by TEM. As the strain is increased to a level of 0.3 , the peak is dissolved in favour of a distribution of entirely different character, characterized by a broad peak situated at some $150 \mu \mathrm{m}$ off the interface to one side.

This change in shape is accompanied by an evolution of the crystallographic character of the interface, and changes in the Burgers vector and line direction components of the GND field. It is evident from figures 10 and 11 that there has been a reduction in the fraction of dislocations with the Burgers vectors lying in the plane of the interface on the high Taylor factor side (crystal A) as the strain increases. The opposite is true on the low Taylor factor side (crystal B). Figures 12 and 13 (and the plots for other components of the dislocation tensor field) indicate a tendency for dislocations with line components perpendicular to the inter- 
face to increase more in strength with increasing strain level than dislocations with line components lying in the plane of the interface.

Based upon the experimental evidence presented here, the exact role of the interface and its influence on the surrounding GND field is not clear. Given that the peak in the distribution at 0.1 strain is higher than the plateau level in crystal $\mathrm{B}$ at 0.3 strain, it would seem that the interface has absorbed dislocations (i.e. exhibiting sink behaviour at higher strain levels). However, many of the observed features can also be explained by an interface which acts as a source for lattice dislocations, assisting in the production of the surrounding GND distribution and simultaneously altering its character. Emission of dislocations has been documented in both coincident site lattice (CSL) boundaries (Lee et al. 1990) and at general boundaries (Murr 1981, Kurzydlowski et al. 1984). Indirect evidence by slip propagation across grain boundaries has been documented by many investigators (Lim and Raj 1985). However, the emission and absorption of dislocations are not considered within the framework of classical continuum dislocation theory, nor can these processes be distinguished by the mesoscale experimental methods described in this paper.

The observations highlighted in figures 14 and 15 suggest that the bicrystal interface is an active site for orientation change. When viewed from a distance (far field) the boundary would appear to have consistent character, but locally (near field) the rather broad dispersion of misorientation suggests a complex structure and character to the interface. The association of some components of the near-field boundary with $\Sigma=15$ may suggest an energy influence on the evolving GND distribution adjacent to the interface. That the excess free energy of some low- $\Sigma$ CSL boundaries is reduced relative to the energy of large-angle random boundaries is well established for high-purity aluminium (Otsuki and Mizuno 1986). If the evolving interface can restructure itself with plastic deformation to incorporate higher fractions of low energy boundaries, we might expect to see an increase in the area fraction of interfaces which have CSL character in conjunction with increasing intragranular structure (e.g. small-angle grain boundaries). Although such increases have been reported for other alloy systems in connection with plastic deformation and subsequent annealing (Palumbo et al. 1992), these changes have heretofore been attributed to twinning processes. The mechanism of evolution contemplated here does not require twinning to increase the CSL density, only alterations in the intragranular GND distribution.

The observations of this work are consistent with the modelling and simulation of microyielding in polycrystalline materials (Meyers and Ashworth 1982). It has been proposed that elastic anisotropy of adjacent grains establishes localized stress concentrations near grain boundaries. These concentrations result in a thin layer of microyielding, and in the generation of GNDs in the vicinity of the boundaries. Work hardening associated with this layer is presumed to account for the well known Hall-Petch effect on yielding. The complex nature of the GND distribution near the bicrystalline interface suggests that substantial differences may be expected in the interaction of the interface with deformation as a function of interface character. Thus, one might expect the Hall-Petch relationship to have dependence upon the grain-boundary character distribution.

\section{ACKNOWLEDGEMENTS}

This work was partially supported by MRSEC Program of the National Science Foundation (DMR-9632556), and under the auspices of the US Department 
of Energy and Lawrence Livermore National Laboratory under contract W-7405Eng-48. S. Sun gratefully acknowledges the comments of Professor E. T. Onat, Professor B. T. Chu and Professor W. Tong of the Mechanical Engineering Department at Yale University, and their valuable guidance in the process of completing this project as a doctoral dissertation. The assistance of B. El-Dasher of Carnegie-Mellon University is also gratefully acknowledged.

\section{REFERENCES}

Adams, B. L., Wright, S. I., and Kunze, K., 1993, Metall. Trans. A, 24, 819.

Ashby, M. F., 1970, Phil. Mag. 21, 339.

Barlow, C. Y., Bay, B., and Hansen, N., 1985, Phil. Mag. A, 51, 253.

BeCKer, R., 1991, Acta metall. mater., 39, 1211; 1995, Modeling Simul. Mater. Sci. Engng., 3, 417.

Bilby, B. A, Gardner, L. R. T., and Sirth, E., 1958, Acta metall., 6, 29.

Brandon, D. G., 1966, Acta metall., 14, 1479.

Dantz IG, G. B., 1963, Linear Programming and Extensions (Princeton University Press).

Fleck, N. A., Muller, G. M., Ashby, M. F., and Hutchinson, J. W., 1994, Acta metall. mater., 42, 475.

Harren, S. V., Deve, H. E., and Asaro, R. J., 1988, Acta metall., 36, 2435.

KING, W., 1991, Energy and Technology: Review (Livermore, California: Lawrence Livermore National Laboratory), p. 218.

KröNer, E., 1958, Continuum Theory of Dislocations and Self Stresses (Berlin: Springer).

Kurzydlowski, K. J., Varin, R. A., and Zielinski, W., 1984, Acta metall., 32, 71.

Lee, T. C., Robertson, I. M., and Birnbaum, H. K., 1990, Metall. Trans. A, 21, 2437.

Lim, L. C., and RaJ, R., 1985, Acta metall., 33, 1577, 2205.

Lu, Q., 1994, J. appl. Crystallogr., 27, 755.

Meyers, M. A., and Ashworth, E., 1982, Phil. Mag. A, 46, 737.

Murr, L. E., 1981, Mat. Sci. Engng., 51, 71.

NYE, J. F., 1953, Acta metal., 1, 153.

Otsuki, A., and Mizuno, M., 1986, Trans. Japan Inst. Metals, Suppl., 27, 789.

Palumbo, G., Aust, K. T., King, P. J., Brennenstuhl, A. M., and Lichtenberger, P. C., 1992, Phys. St., sol. (a), 131, 425.

Randle, V., Hansen, N., and Juul-Jensen, D., 1996, Phil. Mag. A, 73, 265.

Sun, S., Adams, B. L., Shet, C., and Saigal, S., 1999 (in preparation).

Sutton, A., and Balluffi, R., 1995, Interfaces in Crystalline Materials (Oxford: Clarendon), p. 737.

TAYloR, G. I., 1938, J. Inst. Metals, 68, 307. 